\title{
Passive and smart mechanical behaviour of auxetic open cell PU foams
}

\author{
F Scarpa
}

*Department of Aerospace Engineering, University of Bristol, BS8 1TR Bristol, UK

We present theoretical and experimental results on negative Poisson's ratio open cell foams, monophase (i.e, only PU core [1]) and multiphase (skeleton core, solvent, magnetorheological particles [2]). Novel generation passive auxetic PU foams have been manufactured, and their compressive properties measured and simulated via a new modeling technique taking into account single cell properties and statistical distributions of their locations across the mould samples. Tensile and compressive fatigue properties (SN curves) are derived for auxetic and conventional foams under different failure criteria. As example of smart mechanical properties, we describe also the shape memory effect that auxetic foams exhibits (i.e., the return to the original conventional phase). Considerations on the mechanical properties of smart MRF foams are also made using a modified Gent's and 3D Mendel model [3], and experimental results are also presented on stiffness calculations and evidence of active control under external magnetic fields.

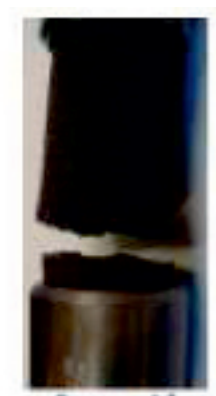

Auxetic

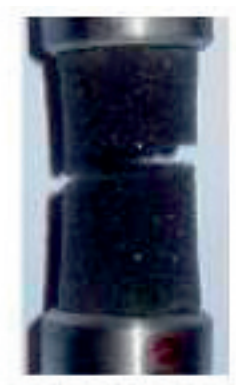

Conventional
Fig. 1 Auxetic and conventional foam specimens after tensile loading failure

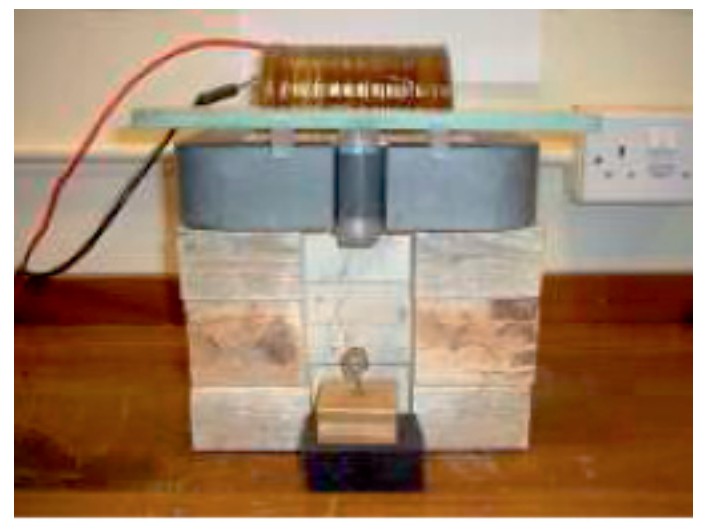

\section{References}

[1]. Scarpa F, Ciffo LG, Yates JR Smart Materials \& Structures 13(1) (2004): 49-56

[2]. Bullough, W. A. and Scarpa, F., 2005. Int. J. Mod. Physics B, 19(7-9), 1656-1665

[3]. Kurashige M, Sato K, Imai K Acta Mechanica 175(1-4) (2005): 25-43 